Article

\title{
Development of Circularly Polarized Luminescence (CPL) Peptides Containing Pyrenylalanines and 2-Aminoisobutyric Acid
}

\author{
Yuki Mimura, Yuki Motomura, Mizuki Kitamatsu * and Yoshitane Imai * \\ Department of Applied Chemistry, Faculty of Science and Engineering, Kindai University, 3-4-1 Kowakae, \\ Higashi-Osaka, Osaka 577-8502, Japan; appletea0424@gmail.com (Y.M.); motomurayuki0818@gmail.com (Y.M.) \\ * Correspondence: kitamatu@apch.kindai.ac.jp (M.K.); y-imai@apch.kindai.ac.jp (Y.I.)
}

Received: 31 October 2020; Accepted: 25 November 2020; Published: 27 November 2020

\begin{abstract}
Chiral organic and organometallic luminophores that possess circularly polarized luminescence (CPL) properties in the near-ultraviolet to near-infrared region have several useful applications. However, the CPL properties are subject to inherent factors of the compounds; to date, studies on the CPL properties influenced by amino acids and peptides are scarce. Consequently, we developed peptide-pyrene organic luminophores exhibiting various CPL properties. It is conceivable that the peptide-pyrene organic luminophores can be obtained as aggregates when dissolved in a solution. It is also possible that the formation of aggregates makes it difficult to accurately examine the CPL of the peptide in the solution. This study showed that the introduction of sterically hindered 2-aminoisobutyric acid (Aib) units into the peptide backbone inhibits aggregate formation. The resulting luminophores exhibit CPL properties owing to the presence of pyrene units. The results of this study can form a basis for the design of future materials that use peptide-pyrene organic luminophores.
\end{abstract}

Keywords: chiral; circularly polarized luminescence (CPL); pyrene; peptide

\section{Introduction}

It has been recently proposed that chiral organic and organometallic luminophores with circularly polarized luminescence (CPL) properties in the near-ultraviolet to near-infrared region can be employed in a variety of useful applications, such as 3D displays and photonic devices (e.g., organic LEDs) [1-11]. However, very few studies have investigated the effects of amino acids and peptides on the CPL properties. We previously investigated peptide-pyrene organic luminophores exhibiting CPL properties where no aggregate formation took place between pyrene units and found that chiral oligopeptides with multiple pyrene units exhibit a strong CPL signal from the excimers. However, it is possible that the pyrenes may form aggregates. In fact, agglomerates of pyrene have been observed when the number of pyrene moieties is increased. Therefore, it is difficult to purify a peptide containing five or more pyrene moieties due to such aggregate formation. To address this issue, we investigate various methods to prevent the formation of aggregates. In this work, we introduce a sterically hindered 2-aminoisobutyric acid (Aib) spacer between the pendant pyrenyl groups to inhibit aggregate formation. In addition, we synthesize an optically active Aib peptide-pyrene luminophore that increases the number of pyrene moieties incorporated into a single peptide (Figure 1a): (1) D-Aib1, (2) D-Aib2, (3) D-Aib3, (4) D-Aib4, (5) D-Aib5, and their corresponding L-isomers (L-Aib1-L-Aib5). The CPL properties of these peptides in a chloroform $\left(\mathrm{CHCl}_{3}\right)$ solution are then investigated. Furthermore, we compare the glycine-containing Gly peptide, which contains more flexible units than the Aib 
(Figure 1b) with previously reported peptide-pyrene luminophores containing two to four pyrene moieties (Figure 1c) [12].
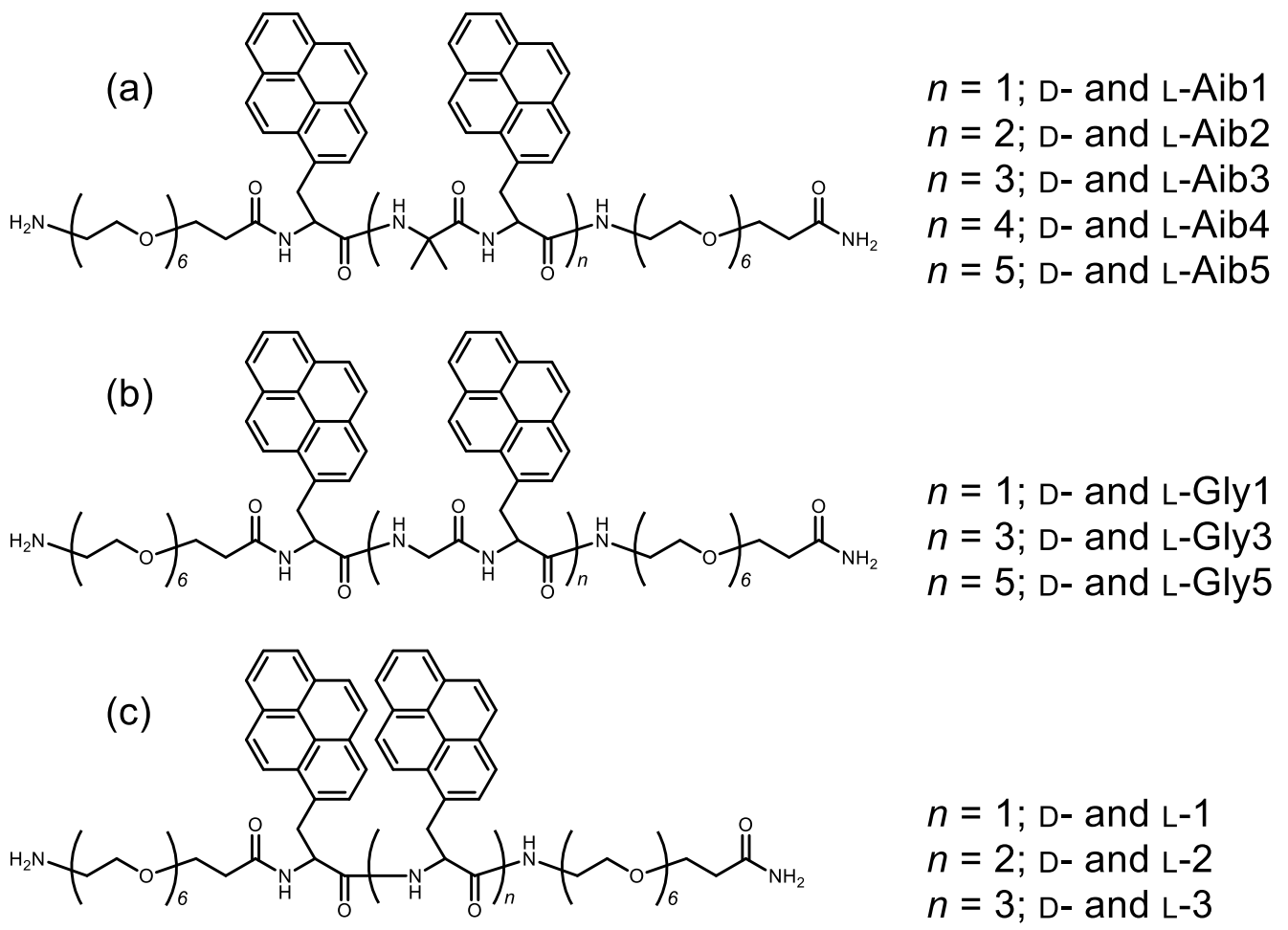

Figure 1. (a) Chiral 2-aminoisobutyric acid (Aib)-pyrenyl oligopeptide luminophores D-Aib1-D-Aib5 and L-Aib1-L-Aib5. (b) Chiral Gly-pyrenyl oligopeptide luminophores D-Gly1, D-Gly3, D-Gly5, and their L-isomers. (c) Chiral pyrenyl oligopeptide luminophores D-1-D-3 and L-1-L-3.

\section{Materials and Methods}

\subsection{Synthesis of Chiral Aib-Pyrenyl Oligopeptides}

The chiral Aib-pyrenyl oligopeptides were prepared by using previously reported conventional 9-fluorenylmethyloxycarbonyl group (Fmoc)-based solid-phase peptide synthesis [12]. The peptides were synthesized using L-pyrenyl alanine (L-Ala(Pyr); Fmoc-Ala(1-Pyn)-OH), D-pyrenyl alanine (D-Ala(Pyr): Fmoc-D-Ala(1-Pyn)-OH), Aib (Fmoc-NH-C( $\left.\left.\mathrm{CH}_{3}\right)_{2}-\mathrm{OH}\right)$, and an amino acid consisting of six ethylene glycol units (Sp6: Fmoc-NH-PEG $6-\mathrm{COOH}$ ) as monomer units. Fmoc-Ala(1-Pyn)-OH, Fmoc-D-Ala(1-Pyn)-OH, and Fmoc-NH-C $\left(\mathrm{CH}_{3}\right)_{2}-\mathrm{OH}$ were purchased from Watanabe Chemicals (Hiroshima, Japan). Fmoc-NH-PEG 6 -COOH was purchased from Merck (Darmstadt, Germany). The Sp6 linker was used for improving the affinity of the peptides against various solvents. The synthesized peptides exhibited the following sequences: H-Sp6-D-Ala(Pyr)-Aib-D-Ala(Pyr)-Sp6-NH 2 (D-Aib1), H-Sp6-(D-Ala(Pyr)-Aib) 2-D-Ala(Pyr)-Sp6-NH2 (D-Aib2), H-Sp6-(D-Ala(Pyr)-Aib) 3 -D-Ala(Pyr)-Sp6-NH (D-Aib3), H-Sp6-(D-Ala(Pyr)-Aib) 4 -D-Ala(Pyr)-Sp6-NH 2 (D-Aib4), H-Sp6-(D-Ala(Pyr)-Aib) 5-D-Ala(Pyr)Sp6-NH 2 (D-Aib5), H-Sp6-L-Ala(Pyr)-Aib-L-Ala(Pyr)-Sp6-NH 2 (L-Aib1), H-Sp6-(L-Ala(Pyr)-Aib) $2_{2}^{-}$

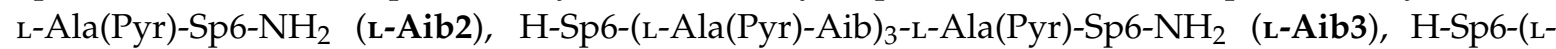
Ala(Pyr)-Aib) ${ }_{4}$-L-Ala(Pyr)-Sp6-NH 2 (L-Aib4), and H-Sp6-(L-Ala(Pyr)-Aib)5-L-Ala(Pyr)-Sp6-NH 2 (L-Aib5).

These peptides were prepared on an Fmoc-NH-super acid-labile poly(ethylene glycol (SAL PEG)) resin (Watanabe Chemicals; $14 \mu \mathrm{mol}$ Fmoc on a resin surface). The deprotection of Fmoc was carried out using $20 \%$ piperidine in $\mathrm{N}, \mathrm{N}^{\prime}$-dimethylformamide (DMF) for $7 \mathrm{~min}$. After washing the peptides six times with $\mathrm{DMF}$, each Fmoc-derivatized amino acid was coupled with $\mathrm{O}-\left(1 \mathrm{H}-\right.$-benzotriazol-1-yl)-N,N,N $\mathrm{N}^{\prime}, \mathrm{N}^{\prime}$-tetramethyluronium hexafluorophosphate (HBTU)/N-methylmorpholine (NMM) in DMF for $50 \mathrm{~min}$. Deprotection and coupling processes were carried out at room temperature without a capping process. The peptides were cleaved from 
the resin by treatment with 95.0:2.5:2.5 (v/v) trifluoroacetic acid (TFA)/water/triisopropylsilane (TIS) for $90 \mathrm{~min}$ at room temperature. Crude peptides were purified by reverse-phase high-performance liquid chromatography (RP-HPLC) on a C18 preparative column (Cadenza 5CD-C18; Imtakt, Kyoto, Japan) using a linear gradient from 35\% to 100\% (D-Aib1 and L-Aib1), from 45\% to 100\% (D-Aib2 and L-Aib2), from 50\% to $100 \%$ (D-Aib3 and L-Aib3), from $60 \%$ to $100 \%$ (D-Aib4 and L-Aib4), and from $85 \%$ to $100 \%$ (D-Aib5 and L-Aib5) of B solvent (acetonitrile; A solvent was $0.1 \%$ TFA ap.) over $30 \mathrm{~min}$ at a flow rate of $10.0 \mathrm{~mL} / \mathrm{min}$ and monitored at $340 \mathrm{~nm}$. The final product was identified using a C18 analytical column (Cadenza CD-C18 (CD003); Imtakt, Kyoto, Japan) and matrix-assisted laser desorption/ionization-time-of-flight (MALDI-TOF) mass spectrometry (Shimadzu AXIMA Confidence) (see Supporting Information: Figures S1-S20). The yields of the peptides were 49\% (D-Aib1), 36\% (D-Aib2), 10\% (D-Aib3), 20\% (D-Aib4), 9\% (D-Aib5), 36\% (L-Aib1), 44\% (L-Aib2), 9\% (L-Aib3), 21\% (L-Aib4), and 6\% (L-Aib5). D-Gly1, D-Gly3, D-Gly5, L-Gly1, L-Gly3, L-Gly5, D-1, D-2, D-3, L-1, $\mathbf{L}-\mathbf{2}$, and $\mathbf{L}-\mathbf{3}$ were synthesized using previously reported methods [13].

\subsection{Measurement of Circularly Polarized Luminescence (CPL) and Photoluminescence (PL) Spectra}

Absolute PL quantum yields in $\mathrm{CHCl}_{3}$ solutions and ethanol solution were obtained using an absolute PL quantum yield measurement system (Hamamatsu Photonics C9920-02, Hamamatsu Photonics, Hamamatsu, Japan) under air at room temperature. Luminophores 1-3 and Aib1-Aib5, each at a concentration of $1.0 \times 10^{-4} \mathrm{M}$, in $\mathrm{CHCl}_{3}$ solutions, were excited at 340 and $300 \mathrm{~nm}$. Luminophores 1-3 and Aib1-Aib5, each at a concentration of $1.0 \times 10^{-4} \mathrm{M}$, in ethanol solutions were excited at $300 \mathrm{~nm}$, respectively. The pass length was $10 \mathrm{~mm}$.

CPL and PL spectra in $\mathrm{CHCl}_{3}$ solutions and ethanol solution were measured using a CPL-300 spectrofluoropolarimeter (JASCO, Tokyo, Japan) at room temperature. The instrument uses a scattering angle of $0^{\circ}$ from the excitation of unpolarized, monochromated incident light with a bandwidth of $10 \mathrm{~nm}$ and a bandwidth for emission of $10 \mathrm{~nm}$. The scanning speed was $50 \mathrm{~nm} \mathrm{~min}{ }^{-1}$ and the time constant of PMT (photomultiplier tube) was $8 \mathrm{~s}$. The CPL and PL spectra were smoothed by two accumulations without any numerical smoothing. Luminophores 1-3 at a concentration of $1.0 \times 10^{-4} \mathrm{M}_{\text {in }} \mathrm{CHCl}_{3}$ solutions were excited at $340 \mathrm{~nm}$. Under the same conditions (i.e., concentration of $1.0 \times 10^{-4} \mathrm{M}_{\text {in }} \mathrm{CHCl}_{3}$ solutions), luminophores Aib1-Aib5 and Gly peptides (Gly1, Gly3, and Gly5) were excited at $300 \mathrm{~nm}$. The pass length was $1 \mathrm{~mm}$ in $\mathrm{CHCl}_{3}$. Luminophores 1-3, Aib1-Aib5, and Gly peptides (Gly1, Gly3, and Gly5) were excited at $300 \mathrm{~nm}$. The pass length was $2 \mathrm{~mm}(\mathbf{1}-\mathbf{3}$ and Aib1-Aib5) and $1 \mathrm{~mm}$ (Gly1, Gly3, and Gly5) in ethanol solution.

\subsection{Measurement of Circular Dichroism (CD) and UV-Vis Absorption Spectra}

CD and UV-Vis absorption spectra of Aib1-Aib5 and 1-3 and Gly peptides (Gly1, Gly3, and Gly5) in $\mathrm{CHCl}_{3}$ solutions and ethanol solution $\left(1.0 \times 10^{-4} \mathrm{M}\right)$ were measured using a JASCO $\mathrm{J}-820$ spectropolarimeter at room temperature. The pass length was $1 \mathrm{~mm}$ in $\mathrm{CHCl}_{3}$. In ethanol, the pass length was $2 \mathrm{~mm}$ (1-3 and Aib1-Aib5) and $1 \mathrm{~mm}$ (Gly1, Gly3, and Gly5).

\section{Results and Discussion}

3.1. Measurement of Aib1-Aib5 Circularly Polarized Luminescence (CPL) and Photoluminescence (PL) Spectra and Circular Dichroism (CD) and UV-Vis Absorption Spectra in $\mathrm{CHCl}_{3}$ Solution

The unpolarized photoluminescence (PL) and CPL spectra of D-Aib1-D-Aib5 and L-isomer (L-Aib1-L-Aib5) in $\mathrm{CHCl}_{3}$ solution $\left(1.0 \times 10^{-4} \mathrm{M}\right)$ were measured to investigate the effect of number of pyrenes and Aib units. Although the common problem in organic luminophores is aggregation-induced quenching [14], D-Aib1-D-Aib5 emitted PL from the fluorescent pyrene units in the $\mathrm{CHCl}_{3}$ solution (Figure 2a-e) for D-Aib1-D-Aib5, indicated by the red lines in the lower panels. As anticipated, D-Aib1-D-Aib5 emitted both the monomer and excimer PLs. The excimer PL increased in the order D-Aib1 $<$ D-Aib2 $<$ D-Aib3 $<$ D-Aib4 $<$ D-Aib5. The maximum wavelengths of monomer PL emissions 
$\left(\lambda_{\text {em }}\right)$ for D-Aib1-D-Aib5 were 379, 395.5, 395.5, 395.5, and 395.5 nm (L-isomer: 379, 395.5, 395.5, 395.5, and $395.5 \mathrm{~nm}$, respectively). On the other hand, the corresponding excimer PL emissions $\left(\lambda_{\text {em }}\right)$ were $473.5,460.5,449$, and $451.5 \mathrm{~nm}$, respectively (L-isomer: $474.5,453.5$, 448, and $451.5 \mathrm{~nm}$, respectively). Although the monomer-to-excimer emission intensity ratio increased in the order Aib1 $<$ Aib2 $<$ Aib3 < Aib4 < Aib5, no clear differences between the $\lambda_{\mathrm{em}}$ values of the monomer and excimer PLs were observed. The corresponding PL quantum yields $\left(\Phi_{\mathrm{F}}\right)$ for Aib1-Aib5 were 0.11 , $0.12,0.12,0.14$, and 0.17, respectively. The relatively low $\Phi_{\mathrm{F}}$ values for Aib1-Aib5 result from the flexibility of the peptide backbone in the oligopeptide luminophores.

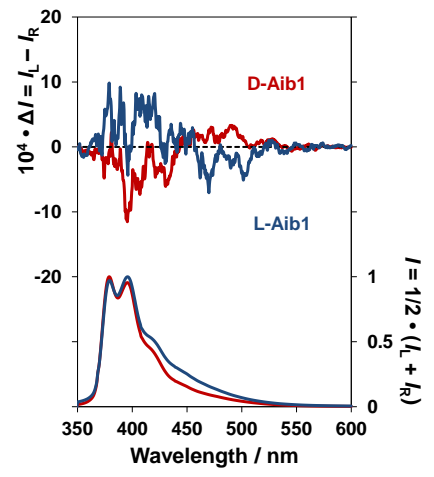

(a)

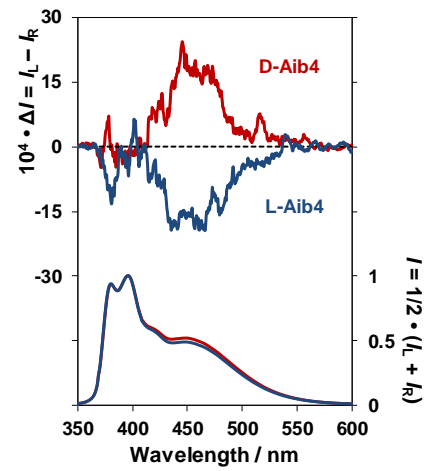

(d)

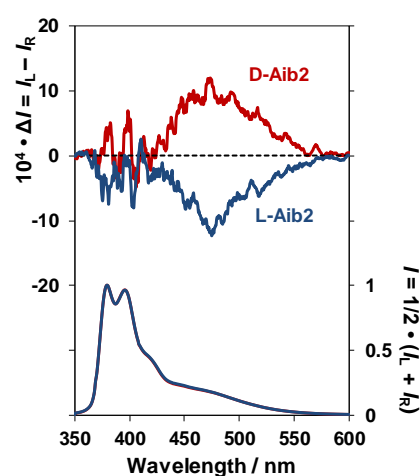

(b)

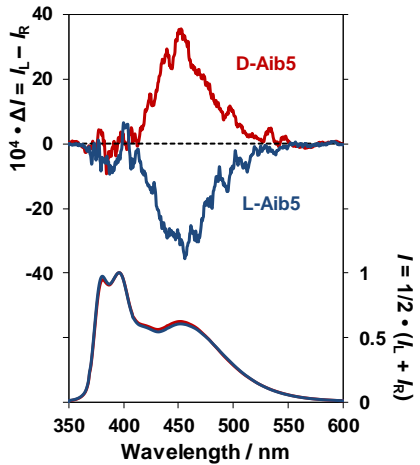

(e)

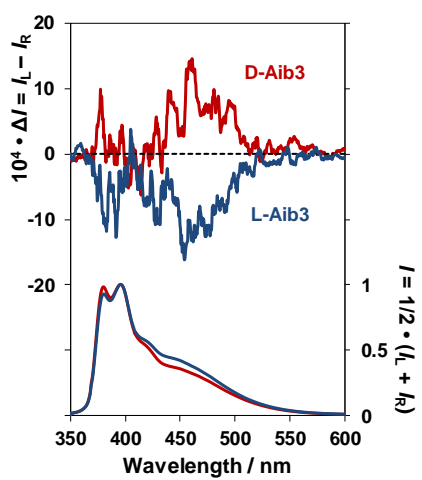

(c)

Figure 2. Circularly polarized luminescence (CPL) (upper panel) and photoluminescence (PL) (lower panel) spectra of (a) D-Aib1/L-Aib1; (b) D-Aib2/L-Aib2; (c) D-Aib3/L-Aib3; (d) D-Aib4/L-Aib4; and $(\mathbf{e}) \mathbf{D}$-Aib5/L-Aib5 in $\mathrm{CHCl}_{3}\left(1.0 \times 10^{-4} \mathrm{M}\right)$. D-isomer and L-isomer spectra are shown in red and blue, respectively. Path length $=1 \mathrm{~mm}$.

The CPL intensity was quantitatively evaluated using the formula, $g_{\mathrm{CPL}}=\Delta \mathrm{I} / \mathrm{I}=\left(I_{\mathrm{L}}-I_{\mathrm{R}}\right) /\left[\left(I_{\mathrm{L}}+I_{\mathrm{R}}\right) / 2\right]$, in which $I_{\mathrm{L}}$ and $I_{\mathrm{R}}$ are the output signal intensities for left- and right-handed circularly polarized light, respectively, under unpolarized photoexcitation conditions. For Aib1, no clear CPL spectra were observed (upper panel of Figure $2 \mathbf{a}$, indicated by the red and blue lines). In addition, the CPL spectra of Aib2-Aib5 in $\mathrm{CHCl}_{3}$ were quite different from that of Aib1, as shown in the upper panels of Figure $2 b-e$ (D-isomer and L-isomer spectra are shown in red and blue, respectively). A strong excimer CPL band was mainly observed for Aib2-Aib5, whereas no or very weak monomer CPL signals were observed. In Aib2-Aib5, as in 1-3 (see reference [12]), the excimer CPL was (+)-CPL in the D-isomer and (-)-CPL in the L-isomer. These CPL spectra were comparable but mirror images of one another. These excimer CPL signals originated from the multiple pyrene units located at distant locations within the molecule of the peptide backbone with the same chirality. The absolute anisotropy factor values for the excimer CPL, $\mathrm{g}_{\mathrm{CPL}}$, were $+6.7 \times 10^{-3}$ (D-isomer) and $-7.0 \times 10^{-3}$ (L-isomer) for Aib2, $+4.6 \times 10^{-3}$ (D-isomer) and $-4.0 \times 10^{-3}$ (L-isomer) for Aib3, $+4.7 \times 10^{-3}$ (D-isomer) and $-4.4 \times 10^{-3}$ (L-isomer) for Aib4, and $+5.7 \times 10^{-3}$ (D-isomer) and $-5.9 \times 10^{-3}$ (L-isomer) for Aib5. The obtained CPL 
and PL properties are summarized in Table 1 together with other key data. The CPL data of Aib1-5 and 1-3 in ethanol solution are included in the Supporting Information (Figures S21a-e and S22a-c). As shown, in ethanol, clear excimer CPL was observed in the cases of Aib1-Aib5. However, only 1 exhibited a clear excimer CPL, with no clear excimer CPL being observed for 2 and 3 . In the cases of Aib1-Aib5, no aggregates were observed when dissolved in ethanol. However, in the case of 3, small aggregates were detected. The CPL data obtained in ethanol solution are summarized in the Supporting Information (Table S1).

Table 1. CPL, PL, circular dichroism (CD), and UV-Vis properties of peptide-pyrene luminophores in $\mathrm{CHCl}_{3}$.

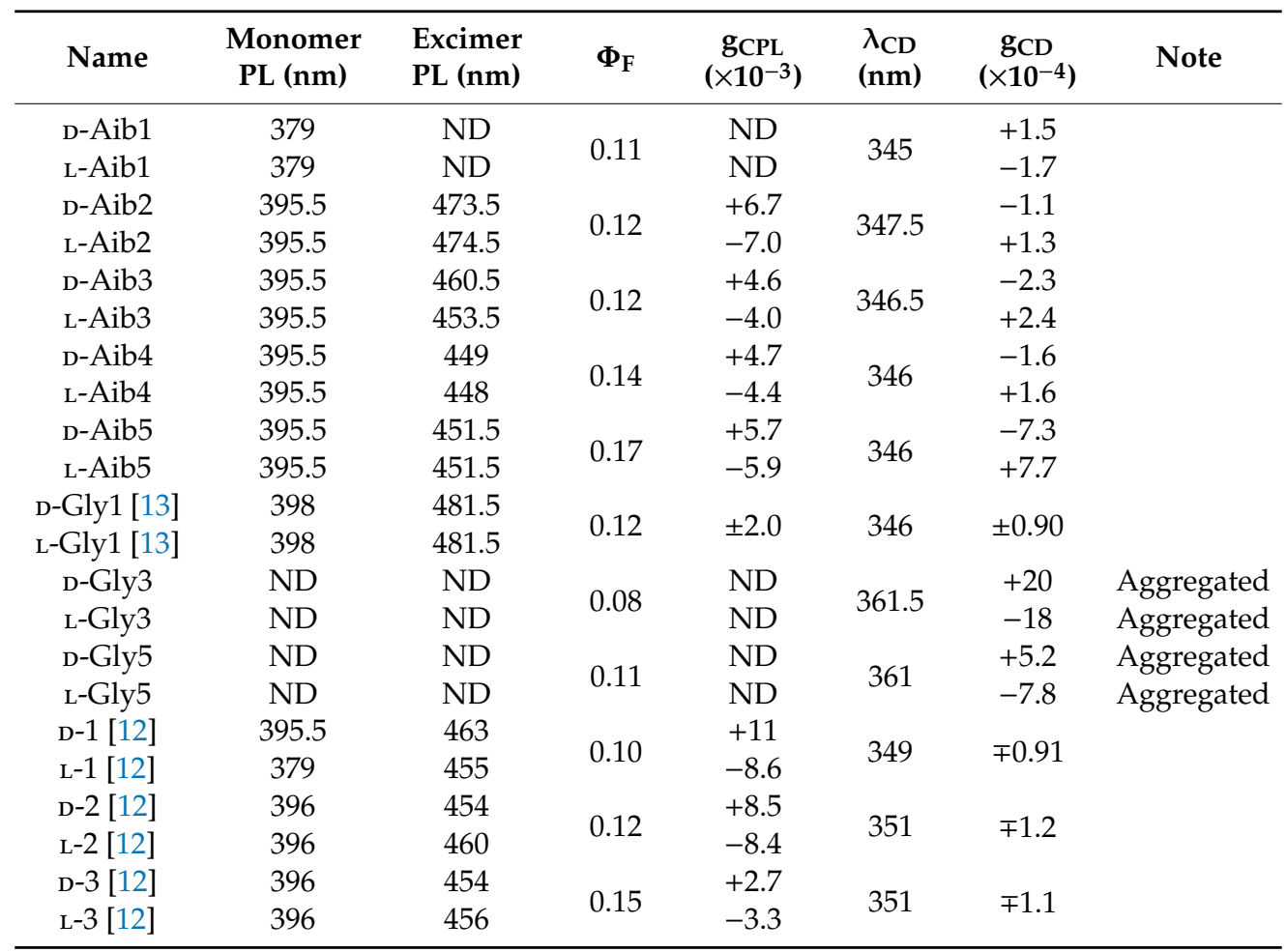

To examine the ground-state chiralities of Aib1-Aib5, we compared their CD and UV-Vis absorption spectra in the $\mathrm{CHCl}_{3}$ solution (Figure $3 \mathrm{a}-\mathrm{e}$ ). The $\lambda_{\mathrm{CD}}$ values of $\mathbf{D}$-Aib1-D-Aib5 were as follows: $345,347.5,346.5,346$, and $346 \mathrm{~nm}$, respectively, while the $\mathrm{g}_{\mathrm{CD}}$ values of ground-state chirality [15] were $+1.5 \times 10^{-4}$ (D-isomer) and $-1.7 \times 10^{-4}$ (L-isomer) for Aib1, $-1.1 \times 10^{-4}$ (D-isomer) and $+1.3 \times 10^{-4}$ (L-isomer) for Aib2, $-2.3 \times 10^{-4}$ (D-isomer) and $+2.4 \times 10^{-4}$ (L-isomer) for Aib3, $-1.6 \times 10^{-4}$ (D-isomer) and $+1.6 \times 10^{-4}$ (L-isomer) for Aib4, and $-7.3 \times 10^{-4}$ (D-isomer) and $+7.7 \times 10^{-4}$ (L-isomer) for Aib5. Interestingly, D-Aib1 exhibited a positive (+) first Cotton band, while D-Aib2-D-Aib5 gave negative (-) Cotton bands. In addition, monomer CPL was observed for Aib1, but clear excimer CPL was observed for Aib2-Aib5. Furthermore, the sign of $\lambda_{C D}$ was inverted between Aib1 and Aib2-Aib5. The difference in the Cotton CD band at approximately $350 \mathrm{~nm}$ derived from the pyrene unit suggests the occurrence of excimer CPL. The obtained CD and UV-Vis properties are summarized in Table 1 together with other key data. The CD data of Aib1-5 and 1-3 in ethanol are provided in the Supporting Information (Figures S23a-e and S24a-c). The CD data obtained in ethanol solution are summarized in the Supporting Information (Table S1). 


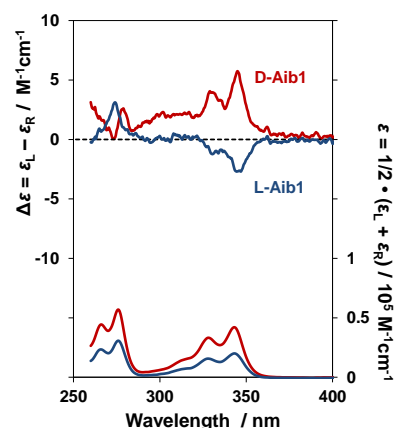

(a)

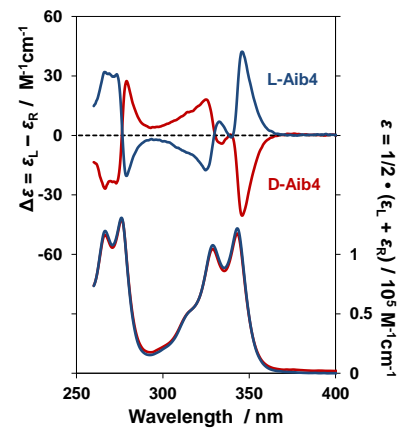

(d)

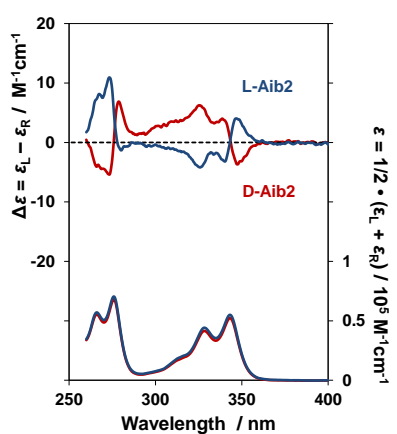

(b)

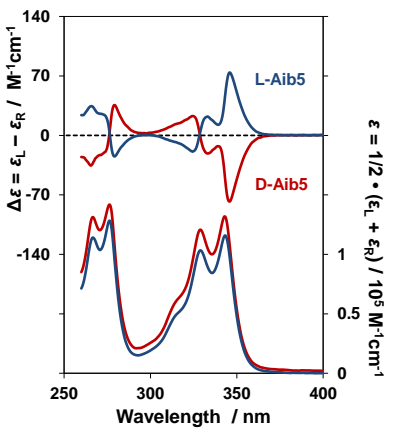

(e)

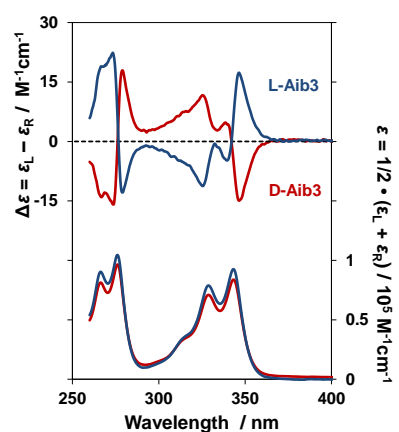

(c)

Figure 3. CD (upper panel) and UV-Vis (lower panel) spectra of (a) D-Aib1/L-Aib1; (b) D-Aib2/L-Aib2; (c) D-Aib3/L-Aib3; (d) D-Aib4/L-Aib4; and (e) D-Aib5/L-Aib5 in $\mathrm{CHCl}_{3}\left(1.0 \times 10^{-4} \mathrm{M}\right)$. D-isomer and L-isomer spectra are shown by red and blue lines, respectively. Path length $=1 \mathrm{~mm}$.

3.2. Measurement of Gly1, Gly3, and Gly5 Circularly Polarized Luminescence (CPL) and Photoluminescence (PL) Spectra and Circular Dichroism (CD) and UV-Vis Absorption Spectra in $\mathrm{CHCl}_{3}$ Solution

The CPL spectra of the Gly units exhibiting less steric hindrance (i.e., Gly1, Gly3, and Gly5) in the $\mathrm{CHCl}_{3}$ solution are shown in Figure $4 \mathrm{a}-\mathrm{c}$. In the case of Gly1, monomer CPL and excimer CPL from the pyrene unit were both observed. However, no CPL characteristics were observed for Gly3 and Gly5, where the formation of aggregates was observed when mixed with $\mathrm{CHCl}_{3}$. Indeed, the $\mathrm{CHCl}_{3}$ solution was a cloudy suspension that did not transmit sufficient light. It is possible that aggregate formation in Gly1, Gly3, and Gly5 results in their solution concentrations being too low to allow sufficient CPL detection. The obtained CPL and PL properties are summarized in Table 1 together with other key data. The CPL data of Gly1, Gly3, and Gly) in ethanol are provided in the Supporting Information (Figure S25a-c). It was found that aggregate formation also took place in the cases of Gly3 and Gly5 in the ethanol solution. The CPL data obtained in ethanol solution are summarized in the Supporting Information (Table S1).

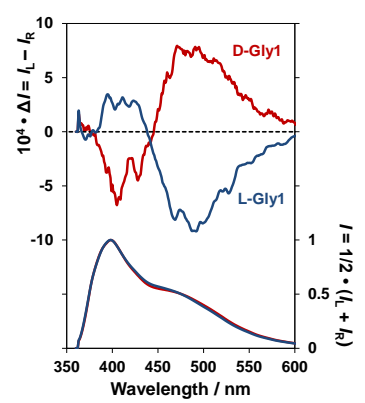

(a)

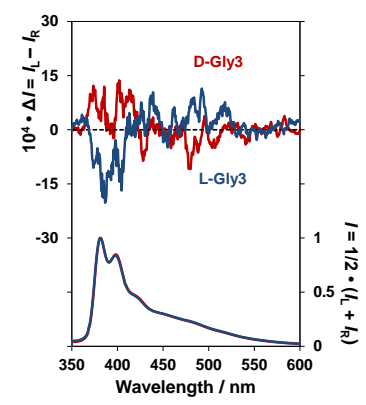

(b)

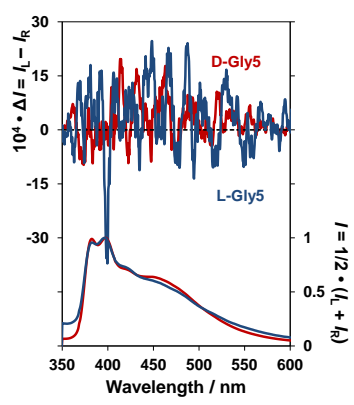

(c)

Figure 4. CPL (upper panel) and PL (lower panel) spectra of (a) D-Gly1/L-Gly1 [13]; (b) D-Gly3/L-Gly3; (c) D-Gly5/L-Gly5 in $\mathrm{CHCl}_{3}\left(1.0 \times 10^{-4} \mathrm{M}\right)$. D-isomer and L-isomer spectra are shown in red and blue, respectively. Path length $=1 \mathrm{~mm}$. 
To examine the ground-state chiralities of Gly1, Gly3, and Gly5, we compared their CD and UV-Vis absorption spectra in the $\mathrm{CHCl}_{3}$ solution (Figure 5a-c). In the case of Gly1, it was observed in the UV-Vis spectra as well as in those of Aib1 and 1, but was hardly detected in the cases of Gly3 and Gly5. It was therefore considered that their CPL spectra exhibit the same phenomenon. In the Gly peptide, the degree of freedom of the peptide increased when the number of Gly units and pyrene units was increased, as the likelihood of aggregate formation between the pyrene units increased. The obtained CD and UV-Vis properties are summarized in Table 1 together with other key data. The CD data of Gly1, Gly3, and Gly5 in the ethanol solution are provided in the Supporting Information (Figure S26a-c).

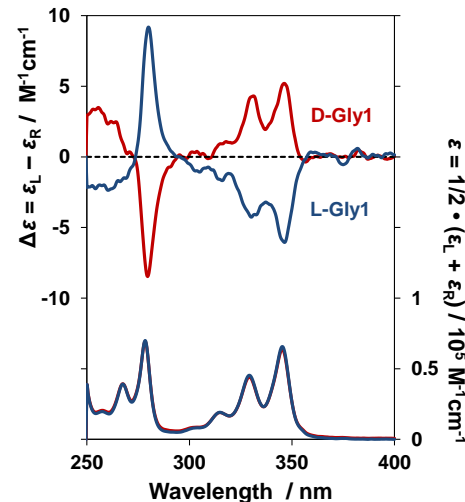

(a)

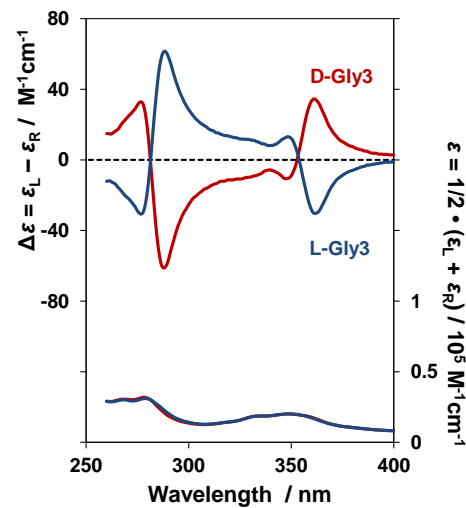

(b)

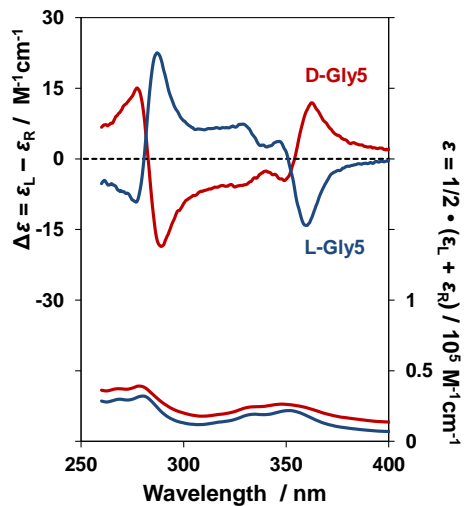

(c)

Figure 5. CD (upper panel) and UV-Vis (lower panel) spectra of (a) D-Gly1/L-Gly1 [13]; (b) D-Gly3/L-Gly3; (c) D-Gly5/L-Gly5 in $\mathrm{CHCl}_{3}\left(1.0 \times 10^{-4} \mathrm{M}\right)$. D-isomer and L-isomer spectra are shown in red and blue, respectively. Path length $=1 \mathrm{~mm}$.

\section{Conclusions}

We developed peptide-pyrene organic luminophores containing the Aib unit. The Aib peptide exhibited excimer CPL in both chloroform and ethanol solutions upon increasing the number of pyrene units. In the ethanol solution, the compounds bearing no Aib unit exhibited trace aggregate formation when four pyrene units were introduced into the peptide. However, it was observed that upon introduction of the Aib unit, no aggregate formation occurred, even when the number of pyrenes was increased to six. Furthermore, when the Gly unit was introduced, the aggregate formation was observed in both chloroform and ethanol solutions when four pyrene units were introduced. Therefore, it was found that there is a high possibility that aggregates will be formed simply by increasing the distance between the pyrene units. In contrast to the Gly peptide, the steric hindrance of the Aib unit inhibits the formation of aggregates, and the number of Gly units can also be adjusted to regulate aggregate formation. We believe that our results may be of assistance in designing future materials using peptide-pyrene organic luminophores.

Supplementary Materials: The following are available online at http://www.mdpi.com/2227-9717/8/12/1550/s1, Figure S1-S10: MALDI-TOF mass spectrum of Aib peptides. Figure S11-S20: RP-HPLC chart of Aib peptides. Figure S21: CPL and PL spectra of Aib1-Aib5 in ethanol. Figure S22: CPL and PL spectra of 1-3 in ethanol. Figure S23: CD and UV-Vis spectra of Aib1-5 in ethanol. Figure S24: CD and UV-Vis spectra of 1-3 in ethanol. Figure S25: CPL and PL spectra of Gly1, Gly3, and Gly5 in ethanol. Figure S26: CD and UV-Vis spectra of Gly1, Gly3, and Gly5 in ethanol.

Author Contributions: Conceptualization, Y.I. and M.K.; methodology, Y.I. and M.K.; software, Y.I.; validation, Y.M. (Yuki Mimura) and Y.M. (Yuki Motomura); formal analysis, Y.M. (Yuki Mimura) and Y.M. (Yuki Motomura); investigation, Y.M. (Yuki Mimura) and Y.M. (Yuki Motomura); resources, Y.I. and M.K.; data curation, Y.I. and M.K.; writing-original draft preparation, Y.M. (Yuki Mimura); writing—review and editing, Y.I. and M.K.; visualization, Y.I. and Y.M. (Yuki Mimura); supervision, Y.I.; project administration, Y.I. and M.K.; funding acquisition, Y.I. All authors have read and agreed to the published version of the manuscript. 
Funding: This study was supported by Grants-in-Aid for Scientific Research (18K05094, 19H02712, 19H04600, and 20H04678) from MEXT/Japan Society for the Promotion of Science; the Research Foundation for KDDI (2019-9); the Electrotechnology of Chubu (R-30506); Futaba (2019-6); Murata Science Foundation (H31-007); the Nippon Sheet Glass Foundation for Materials Science and Engineering (H30-4); the Yashima Environment Technology Foundation (2019-No9). This work was also supported by JST, CREST (JPMJCR2001), Japan.

Conflicts of Interest: The authors declare no conflict of interest. The funding sponsors had no role in the design of the study; in the collection, analyses, or interpretation of data; in the writing of the manuscript; nor in the decision to publish the results.

\section{References}

1. Field, J.E.; Muller, G.; Riehl, J.P.; Venkataraman, D. Circularly Polarized Luminescence from Bridged Triarylamine Helicenes. J. Am. Chem. Soc. 2003, 125, 11808-11809. [CrossRef] [PubMed]

2. Maeda, H.; Bando, Y. Recent progress in research on stimuliresponsive circularly polarized luminescence based on $\pi$-conjugated molecules. Pure Appl. Chem. 2013, 85, 1967-1978. [CrossRef]

3. Sanchez-Carnerero, E.M.; Agarrabeitia, A.R.; Moreno, F.; Maroto, B.L.; Muller, G.; Ortiz, M.J.; Moya, S. Circularly Polarized Luminescence from Simple Organic Molecules. Chem. Eur. J. 2015, 21, 13488-13500. [CrossRef] [PubMed]

4. Kumar, J.; Nakashima, T.; Kawai, T. Circularly Polarized Luminescence in Chiral Molecules and Supramolecular Assemblies. J. Phys. Chem. Lett. 2015, 6, 3445-3452. [CrossRef] [PubMed]

5. Longhi, G.; Castiglioni, E.; Kosyoubu, J.; Mazzeo, G.; Sergio, A. Circularly Polarized Luminescence: A Review of Experimental and Theoretical Aspects. Chirality 2016, 28, 696-707. [CrossRef] [PubMed]

6. Sun, Z.; Suenaga, T.; Sarkar, P.; Sato, S.; Kotani, M.; Isobe, H. Stereoisomerism, crystal structures, and dynamics of belt-shaped cyclonaphthylenes. Proc. Natl. Acad. Sci. USA 2016, 113, 8109-8114. [CrossRef] [PubMed]

7. Tanaka, H.; Inoue, Y.; Mori, T. Circularly Polarized Luminescence and Circular Dichroisms in Small Organic Molecules: Correlation between Excitation and Emission Dissymmetry Factors. ChemPhotoChem 2018, 2, 386-402. [CrossRef]

8. Pop, F.; Zigon, N.; Avarvari, N. Main-Group-Based Electro- and Photoactive Chiral Materials. Chem. Rev. 2019, 119, 8435-8478. [CrossRef]

9. Ma, J.-L.; Peng, Q.; Zhao, C.-H. Circularly Polarized Luminescence Switching in Small Organic Molecules. Chem. Eur. J. 2019, 25, 15441-15454. [CrossRef] [PubMed]

10. Ohishi, Y.; Inouye, M. Circularly polarized luminescence from pyrene excimers. Tetrahedron Lett. 2019, 60, 151232. [CrossRef]

11. Gao, J.; Zhang, W.Y.; Wu, Z.G.; Zheng, Y.X.; Fu, D.W. Enantiomorphic Perovskite Ferroelectrics with Circularly Polarized Luminescence. J. Am. Chem. Soc. 2020, 142, 4756-4761. [CrossRef] [PubMed]

12. Nishikawa, T.; Tajima, N.; Kitamatsu, M.; Fujiki, M.; Imai, Y. Circularly polarised luminescence and circular dichroism of L- and D-oligopeptides with multiple pyrenes. Org. Biomol. Chem. 2015, 13, 11426-11431. [CrossRef] [PubMed]

13. Nishikawa, T.; Kitamura, S.; Kitamatsu, M.; Fujiki, M.; Imai, Y. Peptide Magic: Interdistance-Sensitive Sign Inversion of Excimer Circularly Polarized Luminescence in Bipyrenyl Oligopeptides. ChemistrySelect 2016, 4, 831-835. [CrossRef]

14. Yuan, W.Z.; Lu, P.; Chen, S.; Lam, J.W.Y.; Wang, Z.; Liu, Y.; Kwok, H.S.; Ma, Y.; Tang, B.Z. Changing the Behavior of Chromophores from Aggregation-Caused Quenching to Aggregation-Induced Emission: Development of Highly Efficient Light Emitters in the Solid State. Adv. Mater. 2010, 22, 2159-2163. [CrossRef] [PubMed]

15. The absolute CD magnitude using the dimensionless Kuhn's anisotropy factor in the ground state is defined as $\mathrm{g}_{\mathrm{CD}}=\Delta \varepsilon / \varepsilon$.

Publisher's Note: MDPI stays neutral with regard to jurisdictional claims in published maps and institutional affiliations. 